\title{
A Dependency Pair Framework for Innermost Complexity Analysis of Term Rewrite Systems ${ }^{\star}$
}

\author{
Lars Noschinski ${ }^{1}$, Fabian Emmes ${ }^{2}$, and Jürgen Giesl ${ }^{2}$ \\ ${ }^{1}$ Institut für Informatik, TU Munich, Germany \\ ${ }^{2}$ LuFG Informatik 2, RWTH Aachen University, Germany
}

\begin{abstract}
We present a modular framework to analyze the innermost runtime complexity of term rewrite systems automatically. Our method is based on the dependency pair framework for termination analysis. In contrast to previous work, we developed a direct adaptation of successful termination techniques from the dependency pair framework in order to use them for complexity analysis. By extensive experimental results, we demonstrate the power of our method compared to existing techniques.
\end{abstract}

\section{Introduction}

In practice, termination is often not sufficient, but one also has to ensure that algorithms terminate in reasonable (e.g., polynomial) time. While termination of term rewrite systems (TRSs) is well studied, only recently first results were obtained which adapt termination techniques in order to obtain polynomial complexity bounds automatically, e.g., $[2-5,7,9,15,16,19-21,23,27,28]$. Here, $[3,15,16]$ consider the dependency pair (DP) method $[1,10,11,14]$, which is one of the most popular termination techniques for TRSs. ${ }^{3}$ Moreover, [28] introduces a related modular approach for complexity analysis based on relative rewriting.

Techniques for automated innermost termination analysis of term rewriting are very powerful and have been successfully used to analyze termination of programs in many different languages (e.g., Java [25], Haskell [12], Prolog [26]). Hence, by adapting these termination techniques, the ultimate goal is to obtain approaches which can also analyze the complexity of programs automatically.

In this paper, we present a fresh adaptation of the DP framework for innermost runtime complexity analysis [15]. In contrast to $[3,15,16]$, we follow the original DP framework closely. This allows us to directly adapt the several termination techniques ("processors") of the DP framework for complexity analysis. Like [28], our method is modular. But in contrast to [28], which allows to investigate derivational complexity [17], we focus on innermost runtime complexity. Hence, we can inherit the modularity aspects of the DP framework and benefit from its transformation techniques, which increases power significantly.

\footnotetext{
* Supported by the DFG grant GI 274/5-3.

3 There is also a related area of implicit computational complexity which aims at characterizing complexity classes, e.g., using type systems [18], bottom-up logic programs [13], and also using termination techniques like dependency pairs (e.g., [20]).
} 
After introducing preliminaries in Sect. 2, in Sect. 3 we adapt the concept of dependency pairs from termination analysis to so-called dependency tuples for complexity analysis. While the $D P$ framework for termination works on $D P$ problems, we now work on DT problems (Sect. 4). Sect. 5 adapts the "processors" of the DP framework in order to analyze the complexity of DT problems. We implemented our contributions in the termination analyzer AProVE. Due to the results of this paper, AProVE was the most powerful tool for innermost runtime complexity analysis in the International Termination Competition 2010. This is confirmed by our experiments in Sect. 6, where we compare our technique empirically with previous approaches. All proofs can be found in [24].

\section{Runtime Complexity of Term Rewriting}

See e.g. [6] for the basics of term rewriting. Let $\mathcal{T}(\Sigma, \mathcal{V})$ be the set of all terms over a signature $\Sigma$ and a set of variables $\mathcal{V}$ where we just write $\mathcal{T}$ if $\Sigma$ and $\mathcal{V}$ are clear from the context. The arity of a function symbol $f \in \Sigma$ is denoted by $\operatorname{ar}(f)$ and the size of a term is $|x|=1$ for $x \in \mathcal{V}$ and $\left|f\left(t_{1}, \ldots, t_{n}\right)\right|=1+\left|t_{1}\right|+\ldots+\left|t_{n}\right|$. The derivation height of a term $t$ w.r.t. a relation $\rightarrow$ is the length of the longest sequence of $\rightarrow$-steps starting with $t$, i.e., $\operatorname{dh}(t, \rightarrow)=\sup \left\{n \mid \exists t^{\prime} \in \mathcal{T}, t \rightarrow^{n} t^{\prime}\right\}$, cf. [17]. Here, for any set $M \subseteq \mathbb{N} \cup\{\omega\}$, "sup $M$ " is the least upper bound of $M$. Thus, $\operatorname{dh}(t, \rightarrow)=\omega$ if $t$ starts an infinite sequence of $\rightarrow$-steps.

As an example, consider $\mathcal{R}=\{\mathrm{dbl}(0) \rightarrow 0, \mathrm{dbl}(\mathrm{s}(x)) \rightarrow \mathrm{s}(\mathrm{s}(\mathrm{dbl}(x)))\}$. Then $\operatorname{dh}\left(\operatorname{dbl}\left(\mathrm{s}^{n}(0)\right), \rightarrow_{\mathcal{R}}\right)=n+1$, but $\operatorname{dh}\left(\mathrm{dbl}^{n}(\mathrm{~s}(0)), \rightarrow_{\mathcal{R}}\right)=2^{n}+n-1$.

For a TRS $\mathcal{R}$ with defined symbols $\Sigma_{d}=\{\operatorname{root}(\ell) \mid \ell \rightarrow r \in \mathcal{R}\}$, a term $f\left(t_{1}, \ldots, t_{n}\right)$ is basic if $f \in \Sigma_{d}$ and $t_{1}, \ldots, t_{n}$ do not contain symbols from $\Sigma_{d}$. So for $\mathcal{R}$ above, the basic terms are $\mathrm{dbl}\left(\mathrm{s}^{n}(0)\right)$ and $\mathrm{dbl}\left(\mathrm{s}^{n}(x)\right)$ for $n \in \mathbb{N}, x \in \mathcal{V}$. The innermost runtime complexity function $\operatorname{irc}_{\mathcal{R}}$ maps any $n \in \mathbb{N}$ to the length of the longest sequence of $\dot{i}_{\mathcal{R}}$-steps starting with a basic term $t$ with $|t| \leq n$. Here, " $i_{\mathcal{R}}$ " is the innermost rewrite relation and $\mathcal{T}_{B}$ is the set of all basic terms.

Definition 1 ( $\left.\operatorname{irc}_{\mathcal{R}}[\mathbf{1 5}]\right)$. For a TRS $\mathcal{R}$, its innermost runtime complexity function $\operatorname{irc}_{\mathcal{R}}: \mathbb{N} \rightarrow \mathbb{N} \cup\{\omega\}$ is $\operatorname{irc}_{\mathcal{R}}(n)=\sup \left\{\operatorname{dh}\left(t, \dot{i}_{\mathcal{R}}\right)\left|t \in \mathcal{T}_{B},\right| t \mid \leq n\right\}$.

If one only considers evaluations of basic terms, the (runtime) complexity of the dbl-TRS is linear $\left(\operatorname{irc}_{\mathcal{R}}(n)=n-1\right.$ for $\left.n \geq 2\right)$. But if one also permits evaluations starting with $\mathrm{dbl}^{n}(\mathrm{~s}(0))$, the complexity of the dbl-TRS is exponential.

When analyzing the complexity of programs, one is typically interested in (innermost) evaluations where a defined function like $\mathrm{dbl}$ is applied to data objects (i.e., terms without defined symbols). Therefore, (innermost) runtime complexi$t y$ corresponds to the usual notion of "complexity" for programs $[4,5]$. So for any TRS $\mathcal{R}$, we want to determine the asymptotic complexity of the function $\operatorname{irc}_{\mathcal{R}}$.

Definition 2 (Asymptotic Complexities). Let $\mathfrak{C}=\left\{\mathcal{P o l}_{0}, \mathcal{P}_{\text {ol }}, \mathcal{P}_{\text {ol }}, \ldots\right.$, ? $\}$ with the order $\mathcal{P o l}_{0} \sqsubset \mathcal{P}_{1} l_{1} \sqsubset \mathcal{P} l_{2} \sqsubset \ldots \sqsubset$ ?. Let $\sqsubseteq$ be the reflexive closure of $\sqsubset$. For any function $f: \mathbb{N} \rightarrow \mathbb{N} \cup\{\omega\}$ we define its complexity $\iota(f) \in \mathfrak{C}$ as follows: $\iota(f)=$ Pol $_{k}$ if $k$ is the smallest number with $f(n) \in \mathcal{O}\left(n^{k}\right)$ and $\iota(f)=$ ? if there is no such $k$. For any TRS $\mathcal{R}$, we define its complexity $\iota_{\mathcal{R}}$ as $\iota\left(\operatorname{irc}_{\mathcal{R}}\right)$. 
So the dbl-TRS $\mathcal{R}$ has linear complexity, i.e., $\iota_{\mathcal{R}}=\mathcal{P}_{0} l_{1}$. As another example, consider the following TRS $\mathcal{R}$ where "m" stands for "minus".

$$
\begin{aligned}
& \text { Example 3. } \quad \mathrm{m}(x, y) \rightarrow \text { if }(\mathrm{gt}(x, y), x, y) \quad \text { gt }(0, k) \rightarrow \text { false } \quad \mathrm{p}(0) \rightarrow 0 \\
& \text { if (false, } x, y) \rightarrow 0 \quad \operatorname{gt}(\mathrm{s}(n), \mathrm{s}(k)) \rightarrow \operatorname{gt}(n, k)
\end{aligned}
$$

Here, $\iota_{\mathcal{R}}=\mathcal{P}_{o} l_{2}$ (e.g., $\mathrm{m}\left(\mathrm{s}^{n}(0), \mathrm{s}^{k}(0)\right)$ starts evaluations of quadratic length).

\section{Dependency Tuples}

In the DP method, for every $f \in \Sigma_{d}$ one introduces a fresh symbol $f^{\sharp}$ with $\operatorname{ar}(f)$ $=\operatorname{ar}\left(f^{\sharp}\right)$. For a term $t=f\left(t_{1}, \ldots, t_{n}\right)$ with $f \in \Sigma_{d}$ we define $t^{\sharp}=f^{\sharp}\left(t_{1}, \ldots, t_{n}\right)$ and let $\mathcal{T}^{\sharp}=\left\{t^{\sharp} \mid t \in \mathcal{T}\right.$, $\left.\operatorname{root}(t) \in \Sigma_{d}\right\}$. Let $\mathcal{P}$ os $(t)$ contain all positions of $t$ and let $\mathcal{P}_{o s}(t)=\left\{\pi \mid \pi \in \mathcal{P}\right.$ os $\left.(t), \operatorname{root}\left(\left.t\right|_{\pi}\right) \in \Sigma_{d}\right\}$. Then for every rule $\ell \rightarrow r$ with $\mathcal{P o s}_{d}(r)=\left\{\pi_{1}, \ldots, \pi_{n}\right\}$, its dependency pairs are $\ell^{\sharp} \rightarrow r||_{\pi_{1}}^{\sharp}, \ldots, \ell^{\sharp} \rightarrow r||_{\pi_{n}}^{\sharp}$.

While DPs are used for termination, for complexity we have to regard all defined functions in a right-hand side at once. Thus, we extend the concept of weak dependency pairs $[15,16]$ and only build a single dependency tuple $\ell \rightarrow$ $\left[r\left|\mathbb{\pi}_{1}, \ldots, r\right|_{\pi_{n}}^{\sharp}\right]$ for each $\ell \rightarrow r$. To avoid handling tuples, for every $n \geq 0$, we introduce a fresh compound symbol $\mathrm{CoM}_{n}$ of arity $n$ and use $\ell^{\sharp} \rightarrow \operatorname{CoM}_{n}\left(\left.r\right|_{\pi_{1}} ^{\sharp}, \ldots,\left.r\right|_{\pi_{n}} ^{\sharp}\right)$.

Definition 4 (Dependency Tuple). A dependency tuple is a rule of the form $s^{\sharp} \rightarrow \operatorname{Com}_{n}\left(t_{1}^{\sharp}, \ldots, t_{n}^{\sharp}\right)$ for $s^{\sharp}, t_{1}^{\sharp}, \ldots, t_{n}^{\sharp} \in \mathcal{T}^{\sharp}$. Let $\ell \rightarrow r$ be a rule with $\mathcal{P o s}_{d}(r)=$ $\left\{\pi_{1}, \ldots, \pi_{n}\right\}$. Then DT $(\ell \rightarrow r)$ is defined ${ }^{4}$ to be $\ell^{\sharp} \rightarrow \mathrm{CoM}_{n}\left(r||_{\pi_{1}}^{\sharp}, \ldots, r||_{\pi_{n}}^{\sharp}\right)$. For a TRS $\mathcal{R}$, let $D T(\mathcal{R})=\{D T(\ell \rightarrow r) \mid \ell \rightarrow r \in \mathcal{R}\}$.

Example 5. For the TRS $\mathcal{R}$ from Ex. 3, DT(R) is the following set of rules.

$$
\begin{aligned}
& \mathrm{m}^{\sharp}(x, y) \rightarrow \mathrm{COM}_{2}\left(\text { if }^{\sharp}(\mathrm{gt}(x, y), x, y), \mathrm{gt}^{\sharp}(x, y)\right)(1) \quad \mathrm{p}^{\sharp}(0) \rightarrow \mathrm{CoM}_{0} \\
& \text { if }^{\sharp}(\text { true, } x, y) \rightarrow \mathrm{Com}_{2}\left(\mathrm{~m}^{\sharp}(\mathrm{p}(x), y), \mathrm{p}^{\sharp}(x)\right) \quad \text { (2) } \quad \mathrm{p}^{\sharp}(\mathrm{s}(n)) \rightarrow \mathrm{CoM}_{0} \\
& \text { if }^{\sharp}(\text { false, } x, y) \rightarrow \mathrm{CoM}_{0} \\
& \text { (3) } \mathrm{gt}^{\sharp}(0, k) \rightarrow \mathrm{CoM}_{0} \\
& \mathrm{gt}^{\sharp}(\mathrm{s}(n), 0) \rightarrow \mathrm{COM}_{0} \\
& \mathrm{gt}^{\sharp}(\mathrm{s}(n), \mathrm{s}(k)) \rightarrow \operatorname{Com}_{1}\left(\mathrm{gt}^{\sharp}(n, k)\right)
\end{aligned}
$$

For termination, one analyzes chains of DPs, which correspond to sequences of function calls that can occur in reductions. Since DTs represent several DPs, we now obtain chain trees. (This is analogous to the path detection in [16]).

Definition 6 (Chain Tree). Let $\mathcal{D}$ be a set of DTs and $\mathcal{R}$ be a TRS. Let T be a (possibly infinite) tree whose nodes are labeled with both a DT from $\mathcal{D}$ and a substitution. Let the root node be labeled with $\left(s^{\sharp} \rightarrow \operatorname{CoM}_{n}(\ldots) \mid \sigma\right)$. Then $T$ is a $(\mathcal{D}, \mathcal{R})$-chain tree for $s^{\sharp} \sigma$ if the following holds for all nodes of $T$ : If a node is labeled with $\left(u^{\sharp} \rightarrow \operatorname{COM}_{m}\left(v_{1}^{\sharp}, \ldots, v_{m}^{\sharp}\right) \mid \mu\right)$, then $u^{\sharp} \mu$ is in normal form w.r.t. $\mathcal{R}$. Moreover, if this node has the children $\left(p_{1}^{\sharp} \rightarrow \operatorname{Com}_{m_{1}}(\ldots) \mid \tau_{1}\right), \ldots,\left(p_{k}^{\sharp} \rightarrow\right.$ $\left.\operatorname{Com}_{m_{k}}(\ldots) \mid \tau_{k}\right)$, then there are pairwise different $i_{1}, \ldots, i_{k} \in\{1, \ldots, m\}$ with

\footnotetext{
${ }^{4}$ To make $D T(\ell \rightarrow r)$ unique, we use a total order $<$ on positions where $\pi_{1}<\ldots<\pi_{n}$.
} 


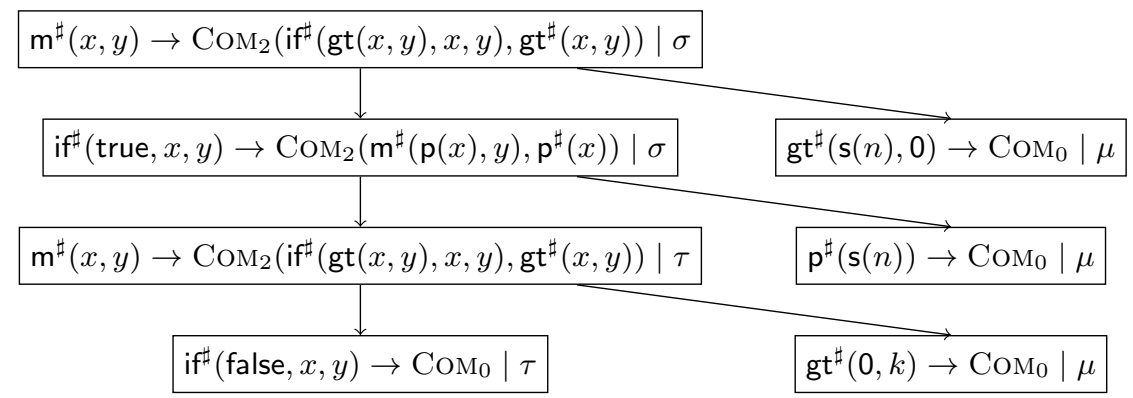

Fig. 1. Chain Tree for the TRS from Ex. 3

$v_{i_{j}}^{\sharp} \mu \stackrel{\mathrm{i}}{\rightarrow}_{\mathcal{R}}^{*} p_{j}^{\sharp} \tau_{j}$ for all $j \in\{1, \ldots, k\}$. A path in the chain tree is called a chain. ${ }^{5}$

Example \%. For the TRS $\mathcal{R}$ from Ex. 3 and its DTs from Ex. 5, the tree in Fig. 1 is a $(D T(\mathcal{R}), \mathcal{R})$-chain tree for $\mathrm{m}^{\sharp}(\mathrm{s}(0), 0)$. Here, we use substitutions with $\sigma(x)=\mathbf{s}(0)$ and $\sigma(y)=0, \tau(x)=\tau(y)=0$, and $\mu(n)=\mu(k)=0$.

For any term $s^{\sharp} \in \mathcal{T}^{\sharp}$, we define its complexity as the maximal number of nodes in any chain tree for $s^{\sharp}$. However, sometimes we do not want to count all DTs in the chain tree, but only the DTs from some subset $\mathcal{S}$. This will be crucial to adapt termination techniques for complexity, cf. Sect. 5.2 and 5.4.

Definition 8 (Complexity of Terms, $\left.\mathcal{C} p l x_{\langle\mathcal{D}, \mathcal{S}, \mathcal{R}\rangle}\right)$. Let $\mathcal{D}$ be a set of dependency tuples, $\mathcal{S} \subseteq \mathcal{D}, \mathcal{R}$ a TRS, and $s^{\sharp} \in \mathcal{T}^{\sharp}$. Then $\mathcal{C} p l x_{\langle\mathcal{D}, \mathcal{S}, \mathcal{R}\rangle}\left(s^{\sharp}\right) \in \mathbb{N} \cup\{\omega\}$ is the maximal number of nodes from $\mathcal{S}$ occurring in any $(\mathcal{D}, \mathcal{R})$-chain tree for $s^{\sharp}$. If there is no $(\mathcal{D}, \mathcal{R})$-chain tree for $s^{\sharp}$, then $\mathcal{C} p l_{\langle\mathcal{D}, \mathcal{S}, \mathcal{R}\rangle}\left(s^{\sharp}\right)=0$.

Example 9. For $\mathcal{R}$ from Ex. 3, we have $\mathcal{C} p l x_{\langle D T(\mathcal{R}), D T(\mathcal{R}), \mathcal{R}\rangle}\left(\mathrm{m}^{\sharp}(\mathrm{s}(0), 0)\right)=7$, since the maximal tree for $\mathrm{m}^{\sharp}(\mathrm{s}(0), 0)$ in Fig. 1 has 7 nodes. In contrast, if $\mathcal{S}$ is $D T(\mathcal{R})$ without the $\mathrm{gt}^{\sharp}-D T \mathrm{~s}(6)-(8)$, then $\mathcal{C} p l x_{\langle D T(\mathcal{R}), \mathcal{S}, \mathcal{R}\rangle}\left(\mathrm{m}^{\sharp}(\mathrm{s}(0), 0)\right)=5$.

Thm. 10 shows how dependency tuples can be used to approximate the derivation heights of terms. More precisely, $\mathcal{C} p l x_{\langle D T(\mathcal{R}), D T(\mathcal{R}), \mathcal{R}\rangle}\left(t^{\sharp}\right)$ is an upper bound for $t$ 's derivation height, provided that $t$ is in argument normal form.

Theorem 10 (Cplx bounds Derivation Height). Let $\mathcal{R}$ be a TRS. Let $t=$ $f\left(t_{1}, \ldots, t_{n}\right) \in \mathcal{T}$ be in argument normal form, i.e., all $t_{i}$ are normal forms w.r.t. $\mathcal{R}$. Then we have $\operatorname{dh}\left(t, \dot{\rightarrow}_{\mathcal{R}}\right) \leq \mathcal{C} p l x_{\langle D T(\mathcal{R}), D T(\mathcal{R}), \mathcal{R}\rangle}\left(t^{\sharp}\right)$. If $\mathcal{R}$ is confluent, we have $\operatorname{dh}\left(t, \dot{\rightarrow}_{\mathcal{R}}\right)=\mathcal{C} p l x_{\langle D T(\mathcal{R}), D T(\mathcal{R}), \mathcal{R}\rangle}\left(t^{\sharp}\right)$.

Note that DTs are much closer to the original DP method than the weak $D P s$ of $[15,16]$. While weak DPs also use compound symbols, they only consider the topmost defined function symbols in right-hand sides of rules. Hence, $[15,16]$ does not use DP concepts when defined functions occur nested on right-hand

\footnotetext{
5 These chains correspond to the "innermost chains" in the DP framework $[1,10,11]$. To handle full (i.e., not necessarily innermost) runtime complexity, one would have to adapt Def. 6 (e.g., then $u^{\sharp} \mu$ would not have to be in normal form).
} 
sides (as in the $\mathrm{m}$ - and the first if-rule) and thus, it cannot fully benefit from the advantages of the DP technique. Instead, $[15,16]$ has to impose several restrictions which are not needed in our approach, cf. Footnote 10. The close analogy of our approach to the DP method allows us to adapt the termination techniques of the DP framework in order to work on DTs (i.e., in order to analyze $\mathcal{C} p l x_{\langle D T(\mathcal{R}), D T(\mathcal{R}), \mathcal{R}\rangle}\left(t^{\sharp}\right)$ for all basic terms $t$ of a certain size). Using Thm. 10, this yields an upper bound for the complexity $\iota_{\mathcal{R}}$ of the TRS $\mathcal{R}$, cf. Thm. 14 . Note that there exist non-confluent $\operatorname{TRSs}^{6}$ where $\mathcal{C} p l x_{\langle D T(\mathcal{R}), D T(\mathcal{R}), \mathcal{R}\rangle}\left(t^{\sharp}\right)$ is exponentially larger than $\operatorname{dh}\left(t, \dot{\rightarrow}_{\mathcal{R}}\right.$ ) (in contrast to $[15,16]$, where the step from TRSs to weak DPs does not change the complexity). However, our main interest is in TRSs corresponding to "typical" (confluent) programs. Here, the step from TRSs to DTs does not "lose" anything (i.e., one has equality in Thm. 10).

\section{DT Problems}

Our goal is to find out automatically how large $\mathcal{C} p l x_{\langle\mathcal{D}, \mathcal{S}, \mathcal{R}\rangle}\left(t^{\sharp}\right)$ could be for basic terms $t$ of size $n$. To this end, we will repeatedly replace the triple $\langle\mathcal{D}, \mathcal{S}, \mathcal{R}\rangle$ by "simpler" triples $\left\langle\mathcal{D}^{\prime}, \mathcal{S}^{\prime}, \mathcal{R}^{\prime}\right\rangle$ and examine $\mathcal{C} p l x_{\left\langle\mathcal{D}^{\prime}, \mathcal{S}^{\prime}, \mathcal{R}^{\prime}\right\rangle}\left(t^{\sharp}\right)$ instead.

This is similar to the DP framework where termination problems are represented by so-called DP problems (consisting of a set of DPs and a set of rules) and where DP problems are transformed into "simpler" DP problems repeatedly. For complexity analysis, we consider "DT problems" instead of "DP problems" (our "DT problems" are similar to the "complexity problems" of [28]).

Definition 11 (DT Problem). Let $\mathcal{R}$ be a TRS, $\mathcal{D}$ a set of $D T s, \mathcal{S} \subseteq \mathcal{D}$. Then $\langle\mathcal{D}, \mathcal{S}, \mathcal{R}\rangle$ is a DT problem and $\mathcal{R}$ 's canonical DT problem is $\langle D T(\mathcal{R}), D T(\mathcal{R}), \mathcal{R}\rangle$.

Thm. 10 showed the connection between the derivation height of a term and the maximal number of nodes in a chain tree. This leads to the definition of the complexity of a $D T$ problem $\langle\mathcal{D}, \mathcal{S}, \mathcal{R}\rangle$. It is defined as the asymptotic complexity of the function $\operatorname{irc}_{\langle\mathcal{D}, \mathcal{S}, \mathcal{R}\rangle}$ which maps any number $n$ to the maximal number of $\mathcal{S}$-nodes in any $(\mathcal{D}, \mathcal{R})$-chain tree for $t^{\sharp}$, where $t$ is a basic term of at most size $n$.

Definition 12 (Complexity of DT Problems). For a DT problem $\langle\mathcal{D}, \mathcal{S}, \mathcal{R}\rangle$, its complexity function is $\operatorname{irc}_{\langle\mathcal{D}, \mathcal{S}, \mathcal{R}\rangle}(n)=\sup \left\{\mathcal{C} p l x_{\langle\mathcal{D}, \mathcal{S}, \mathcal{R}\rangle}\left(t^{\sharp}\right)\left|t \in \mathcal{T}_{B},\right| t \mid \leq n\right\}$. We define the complexity $\iota_{\langle\mathcal{D}, \mathcal{S}, \mathcal{R}\rangle}$ of the DT problem as $\iota\left(\operatorname{irc}_{\langle\mathcal{D}, \mathcal{S}, \mathcal{R}\rangle}\right)$.

Example 13. Consider $\mathcal{R}$ from Ex. 3 and let $\mathcal{D}=D T(\mathcal{R})=\{(1), \ldots,(8)\}$. For $t \in \mathcal{T}_{B}$ with $|t|=n$, the maximal chain tree for $t^{\sharp}$ has approximately $n^{2}$ nodes, i.e., $\operatorname{irc}_{\langle\mathcal{D}, \mathcal{D}, \mathcal{R}\rangle}(n) \in \mathcal{O}\left(n^{2}\right)$. Thus, $\langle\mathcal{D}, \mathcal{D}, \mathcal{R}\rangle$ 's complexity is $\iota_{\langle\mathcal{D}, \mathcal{D}, \mathcal{R}\rangle}=\mathcal{P}_{o} l_{2}$.

Thm. 14 shows that to analyze the complexity of a TRS $\mathcal{R}$, it suffices to analyze the complexity of its canonical DT problem: By Def. $2, \iota_{\mathcal{R}}$ is the complexity of the runtime complexity function $\operatorname{irc}_{\mathcal{R}}$ which maps $n$ to the length of the longest innermost rewrite sequence starting with a basic term of at most size $n$. By Thm. 10 , this length is less than or equal to the size $\mathcal{C} p x_{\langle D T(\mathcal{R}), D T(\mathcal{R}), \mathcal{R}\rangle}\left(t^{\sharp}\right)$ of the max-

\footnotetext{
${ }^{6}$ Consider the TRS $\mathrm{f}(\mathrm{s}(x)) \rightarrow \mathrm{f}(\mathrm{g}(x)), \mathrm{g}(x) \rightarrow x, \mathrm{~g}(x) \rightarrow \mathrm{a}(\mathrm{f}(x))$. Its runtime complex-
} ity is linear, but for any $n>0$, we have $\mathcal{C} p l x_{\langle D T(\mathcal{R}), D T(\mathcal{R}), \mathcal{R}\rangle}\left(f^{\sharp}\left(\mathrm{s}^{n}(0)\right)\right)=2^{n+1}-2$. 
imal chain tree for any basic term $t$ of at most size $n$, i.e., to $\operatorname{irc}_{\langle D T(\mathcal{R}), D T(\mathcal{R}), \mathcal{R}\rangle}(n)$. Theorem 14 (Upper bound for TRSs via Canonical DT Problems). Let $\mathcal{R}$ be a TRS and let $\langle\mathcal{D}, \mathcal{D}, \mathcal{R}\rangle$ be the corresponding canonical DT problem. Then we have $\iota_{\mathcal{R}} \sqsubseteq \iota_{\langle\mathcal{D}, \mathcal{D}, \mathcal{R}\rangle}$ and if $\mathcal{R}$ is confluent, we have $\iota_{\mathcal{R}}=\iota_{\langle\mathcal{D}, \mathcal{D}, \mathcal{R}\rangle}$.

Now we can introduce our notion of processors which is analogous to the "DP processors" for termination $[10,11]$ (and related to the "complexity problem processors" in [28]). A DT processor transforms a DT problem $P$ to a pair $\left(c, P^{\prime}\right)$ of an asymptotic complexity $c \in \mathfrak{C}$ and a DT problem $P^{\prime}$, such that $P^{\prime}$ s complexity is bounded by the maximum of $c$ and of the complexity of $P^{\prime}$.

Definition 15 (Processor, $\oplus$ ). A DT processor Proc is a function $\operatorname{Proc}(P)$ $=\left(c, P^{\prime}\right)$ mapping any DT problem $P$ to a complexity $c \in \mathfrak{C}$ and a DT problem $P^{\prime}$. A processor is sound if $\iota_{P} \sqsubseteq c \oplus \iota_{P^{\prime}}$. Here, " $\oplus$ " is the "maximum" function on $\mathfrak{C}$, i.e., for any $c, d \in \mathfrak{C}$, we define $c \oplus d=d$ if $c \sqsubseteq d$ and $c \oplus d=c$ otherwise.

To analyze the complexity $\iota_{\mathcal{R}}$ of a TRS $\mathcal{R}$, we start with the canonical DT problem $P_{0}=\langle D T(\mathcal{R}), D T(\mathcal{R}), \mathcal{R}\rangle$. Then we apply a sound processor to $P_{0}$ which yields a result $\left(c_{1}, P_{1}\right)$. Afterwards, we apply another (possibly different) sound processor to $P_{1}$ which yields $\left(c_{2}, P_{2}\right)$, etc. This is repeated until we obtain a solved DT problem (whose complexity is obviously $\mathcal{P}_{0} l_{0}$ ).

Definition 16 (Proof Chain, Solved DT Problem). We call a DT problem $P=\langle\mathcal{D}, \mathcal{S}, \mathcal{R}\rangle$ solved, if $\mathcal{S}=\varnothing$. A proof chain ${ }^{7}$ is a finite sequence $P_{0} \stackrel{c_{1}}{\sim} P_{1} \stackrel{c_{2}}{\sim}$ $\ldots \stackrel{c_{k}}{\sim} P_{k}$ ending with a solved DT problem $P_{k}$, such that for all $0 \leq i<k$ there exists a sound processor $\mathrm{PROC}_{i}$ with $\operatorname{PROC}_{i}\left(P_{i}\right)=\left(c_{i+1}, P_{i+1}\right)$.

By Def. 15 and 16, for every $P_{i}$ in a proof chain, $c_{i+1} \oplus \ldots \oplus c_{k}$ is an upper bound for its complexity $\iota_{P_{i}}$. Here, the empty sum (for $i=k$ ) is defined as $\mathcal{P}_{0} l_{0}$.

Theorem 17 (Approximating Complexity by Proof Chain). Let $P_{0} \stackrel{c_{1}}{\sim}$ $P_{1} \stackrel{c_{2}}{\rightarrow} \ldots \stackrel{c_{k}}{\sim} P_{k}$ be a proof chain. Then $\iota_{P_{0}} \sqsubseteq c_{1} \oplus \ldots \oplus c_{k}$.

Thm. 14 and 17 now imply that our approach for complexity analysis is correct.

Corollary 18 (Correctness of Approach). If $P_{0}$ is the canonical DT problem for a TRS $\mathcal{R}$ and $P_{0} \stackrel{c_{1}}{\sim} \ldots \stackrel{c_{k}}{\rightarrow} P_{k}$ is a proof chain, then $\iota_{\mathcal{R}} \sqsubseteq c_{1} \oplus \ldots \oplus c_{k}$.

\section{DT Processors}

In this section, we present several processors to simplify DT problems automatically. To this end, we adapt processors of the DP framework for termination.

The usable rules processor (Sect. 5.1) simplifies a problem $\langle\mathcal{D}, \mathcal{S}, \mathcal{R}\rangle$ by deleting rules from $\mathcal{R}$. The reduction pair processor (Sect. 5.2) removes DTs from $\mathcal{S}$, based on term orders. In Sect. 5.3 we introduce the dependency graph, on which the leaf removal and knowledge propagation processor (Sect. 5.4) are based. Finally, Sect. 5.5 adapts processors based on transformations like narrowing.

\footnotetext{
7 Of course, one could also define DT processors that transform a DT problem $P$ into a complexity $c$ and a set $\left\{P_{1}^{\prime}, \ldots, P_{n}^{\prime}\right\}$ such that $\iota_{P} \sqsubseteq c \oplus \iota_{P_{1}^{\prime}} \oplus \ldots \oplus \iota_{P_{n}^{\prime}}$. Then instead of a proof chain one would obtain a proof tree.
} 


\subsection{Usable Rules Processor}

As in termination analysis, we can restrict ourselves to those rewrite rules that can be used to reduce right-hand sides of DTs (when instantiating their variables with normal forms). This leads to the notion of usable rules. ${ }^{8}$

Definition 19 (Usable Rules $\mathcal{U}_{\mathcal{R}}[\mathbf{1}]$ ). For a TRS $\mathcal{R}$ and any symbol $f$, let $R l_{\mathcal{R}}(f)=\{\ell \rightarrow r \mid \operatorname{root}(\ell)=f\}$. For any term $t, \mathcal{U}_{\mathcal{R}}(t)$ is the smallest set with

- $\mathcal{U}_{\mathcal{R}}(x)=\varnothing$ if $x \in \mathcal{V}$ and

- $\mathcal{U}_{\mathcal{R}}\left(f\left(t_{1}, \ldots, t_{n}\right)\right)=R l_{\mathcal{R}}(f) \cup \bigcup_{\ell \rightarrow r \in R s_{\mathcal{R}}(f)} \mathcal{U}_{\mathcal{R}}(r) \cup \bigcup_{1 \leq i \leq n} \mathcal{U}_{\mathcal{R}}\left(t_{i}\right)$

For any set $\mathcal{D}$ of DTs, we define $\mathcal{U}_{\mathcal{R}}(\mathcal{D})=\bigcup_{s \rightarrow t \in \mathcal{D}} \mathcal{U}_{\mathcal{R}}(t)$.

So for $\mathcal{R}$ and $D T(\mathcal{R})$ in Ex. 3 and $5, \mathcal{U}_{\mathcal{R}}(D T(\mathcal{R}))$ contains just the gt- and the p-rules. The following processor removes non-usable rules from DT problems. ${ }^{9}$

Theorem 20 (Usable Rules Processor). Let $\langle\mathcal{D}, \mathcal{S}, \mathcal{R}\rangle$ be a DT problem. Then the following processor is sound: $\operatorname{PrOC}(\langle\mathcal{D}, \mathcal{S}, \mathcal{R}\rangle)=\left(\mathcal{P}_{0},\left\langle\mathcal{D}, \mathcal{S}, \mathcal{U}_{\mathcal{R}}(\mathcal{D})\right\rangle\right)$.

So when applying the usable rules processor on the canonical DT problem $\langle\mathcal{D}, \mathcal{D}, \mathcal{R}\rangle$ of $\mathcal{R}$ from Ex. 3, we obtain $\left\langle\mathcal{D}, \mathcal{D}, \mathcal{R}_{1}\right\rangle$ where $\mathcal{R}_{1}$ are the gt- and p-rules.

\subsection{Reduction Pair Processor}

Using orders is one of the most important methods for termination or complexity analysis. In the most basic approach, one tries to find a well-founded order $\succ$ such that every reduction step (strictly) decreases w.r.t. $\succ$. This proves termination and most reduction orders also imply some complexity bound, cf. e.g. [7, 17]. However, direct applications of orders have two main drawbacks: The obtained bounds are often far too high to be useful and there are many TRSs that cannot be oriented strictly with standard orders amenable to automation, cf. [28].

Therefore, the reduction pair processor of the DP framework only requires a strict decrease (w.r.t. $\succ$ ) for at least one DP, while for all other DPs and rules, a weak decrease (w.r.t. $\succsim)$ suffices. Then the strictly decreasing DPs can be deleted. Afterwards one can use other orders (or termination techniques) to solve the remaining DP problem. To adapt the reduction pair processor for complexity analysis, we have to restrict ourselves to CoM-monotonic orders. ${ }^{10}$

Definition 21 (Reduction Pair). A reduction pair $(\succsim, \succ)$ consists of a stable monotonic quasi-order $\succsim$ and a stable well-founded order $\succ$ which are compatible

${ }^{8}$ The idea of applying usable rules also for complexity analysis is due to [15], which introduced a technique similar to Thm. 20.

${ }^{9}$ While Def. 19 is the most basic definition of usable rules, the processor of Thm. 20 can also be used with more sophisticated definitions of "usable rules" (e.g., as in [11]).

${ }^{10}$ In [15] "Com-monotonic" is called "safe". Note that our reduction pair processor is much closer to the original processor of the DP framework than [15]. In the main theorem of [15], all (weak) DPs have to be oriented strictly in one go. Moreover, one even has to orient the (usable) rules strictly. Finally, one is either restricted to nonduplicating TRSs or one has to use orderings $\succ$ that are monotonic on all symbols. 
(i.e., $\succsim 0 \succ 0 \succsim \subseteq \succ)$. An order $\succ$ is Com-monotonic iff $\operatorname{Com}_{n}\left(s_{1}^{\sharp}, \ldots, s_{i}^{\sharp}, \ldots, s_{n}^{\sharp}\right) \succ$ $\operatorname{Com}_{n}\left(s_{1}^{\sharp}, \ldots, t^{\sharp}, \ldots, s_{n}^{\sharp}\right)$ for all $n \in \mathbb{N}$, all $1 \leq i \leq n$, and all $s_{1}^{\sharp}, \ldots, s_{n}^{\sharp}, t^{\sharp} \in \mathcal{T}^{\sharp}$ with $s_{i}^{\sharp} \succ t^{\sharp}$. A reduction pair $(\succsim, \succ)$ is CoM-monotonic iff $\succ$ is CoM-monotonic.

For a DT problem $(\mathcal{D}, \mathcal{S}, \mathcal{R})$, we orient $\mathcal{D} \cup \mathcal{R}$ by $\succsim$ or $\succ$. But in contrast to the processor for termination, if a DT is oriented strictly, we may not remove it from $\mathcal{D}$, but only from $\mathcal{S}$. So the DT is not counted anymore for complexity, but it may still be used in reductions. ${ }^{11}$ We will improve this later in Sect. 5.4.

Example 22. This TRS $\mathcal{R}$ shows why DTs may not be removed from $\mathcal{D} .{ }^{12}$

$$
\mathrm{f}(0) \rightarrow 0 \quad \mathrm{f}(\mathrm{s}(x)) \rightarrow \mathrm{f}(\mathrm{id}(x)) \quad \mathrm{id}(0) \rightarrow 0 \quad \text { id }(\mathrm{s}(x)) \rightarrow \mathrm{s}(\mathrm{id}(x))
$$

Let $\mathcal{D}=D T(\mathcal{R})=\left\{\mathrm{f}^{\sharp}(0) \rightarrow \mathrm{Com}_{0}, \mathrm{f}^{\sharp}(\mathrm{s}(x)) \rightarrow \mathrm{Com}_{2}\left(\mathrm{f}^{\sharp}(\mathrm{id}(x)), \mathrm{id}^{\sharp}(x)\right), \mathrm{id}^{\sharp}(0) \rightarrow\right.$ $\left.\mathrm{Com}_{0}, \quad \mathrm{id}^{\sharp}(\mathrm{s}(x)) \rightarrow \mathrm{Com}_{1}\left(\mathrm{id}^{\sharp}(x)\right)\right\}$, where $\mathcal{U}_{\mathcal{R}}(\mathcal{D})$ are just the id-rules. For the $D T$ problem $\left\langle\mathcal{D}, \mathcal{S}, \mathcal{U}_{\mathcal{R}}(\mathcal{D})\right\rangle$ with $\mathcal{S}=\mathcal{D}$, there is a linear polynomial interpretation [.] that orients the first two DTs strictly and the remaining DTs and usable rules weakly: $[0]=0,[\mathrm{~s}](x)=x+1$, $[\mathrm{id}](x)=x,\left[\mathrm{f}^{\sharp}\right](x)=x+1$, $\left[\mathrm{id}^{\sharp}\right](x)=$ $0,\left[\mathrm{Com}_{0}\right]=0,\left[\mathrm{Com}_{1}\right](x)=x,\left[\mathrm{Com}_{2}\right](x, y)=x+y$. If one would remove the first two DTs from $\mathcal{D}$, there is another linear polynomial interpretation that orients the remaining DTs strictly (e.g., by [id $\left.{ }^{\sharp}\right](x)=x+1$ ). Then, one would falsely conclude that the whole TRS has linear runtime complexity.

Hence, the first two DTs should only be removed from $\mathcal{S}$, not from $\mathcal{D}$. This results in $\left\langle\mathcal{D}, \mathcal{S}^{\prime}, \mathcal{U}_{\mathcal{R}}(\mathcal{D})\right\rangle$ where $\mathcal{S}^{\prime}$ consists of the last two DTs. These DTs can occur quadratically often in reductions with $\mathcal{D} \cup \mathcal{U}_{\mathcal{R}}(\mathcal{D})$. Hence, when trying to orient $\mathcal{S}^{\prime}$ strictly and the remaining DTs and usable rules weakly, we have to use a quadratic polynomial interpretation (e.g., $[0]=0,[\mathrm{~s}](x)=x+2$, [id] $(x)=$ $x,\left[\mathrm{f}^{\sharp}\right](x)=x^{2},\left[\mathrm{id}^{\sharp}\right](x)=x+1,\left[\mathrm{Com}_{0}\right]=0,\left[\mathrm{Com}_{1}\right](x)=x,\left[\mathrm{Com}_{2}\right](x, y)=$ $x+y)$. Hence, now we (correctly) conclude that the TRS has quadratic runtime complexity (indeed, $\left.\operatorname{dh}\left(\mathrm{f}\left(\mathrm{s}^{n}(0)\right), \stackrel{i}{\rightarrow}_{\mathcal{R}}\right)=\frac{(n+1) \cdot(n+2)}{2}\right)$.

So when applying the reduction pair processor to $\langle\mathcal{D}, \mathcal{S}, \mathcal{R}\rangle$, we obtain $(c$, $\left.\left\langle\mathcal{D}, \mathcal{S} \backslash \mathcal{D}_{\succ}, \mathcal{R}\right\rangle\right)$. Here, $\mathcal{D}_{\succ}$ are the strictly decreasing DTs from $\mathcal{D}$ and $c$ is an upper bound for the number of $\mathcal{D}_{\succ}$-steps in innermost reductions with $\mathcal{D} \cup \mathcal{R}$.

Theorem 23 (Reduction Pair Processor). Let $P=\langle\mathcal{D}, \mathcal{S}, \mathcal{R}\rangle$ be a DT problem and $(\succsim, \succ)$ be a CoM-monotonic reduction pair. Let $\mathcal{D} \subseteq \succsim \cup \succ, \mathcal{R} \subseteq \succsim$, and $c \sqsupseteq \iota\left(\operatorname{irc}_{\succ}\right)$ for the function $\operatorname{irc}_{\succ}(n)=\sup \left\{\operatorname{dh}\left(t^{\sharp}, \succ\right)\left|t \in \mathcal{T}_{B},\right| t \mid \leq n\right\} .{ }^{13}$ Then the following processor is sound: $\operatorname{Proc}(\langle\mathcal{D}, \mathcal{S}, \mathcal{R}\rangle)=\left(c,\left\langle\mathcal{D}, \mathcal{S} \backslash \mathcal{D}_{\succ}, \mathcal{R}\right\rangle\right)$.

${ }^{11}$ This idea is also used in [28]. However, [28] treats derivational complexity instead of (innermost) runtime complexity, and it operates directly on TRSs and not on DPs or DTs. Therefore, [28] has to impose stronger restrictions (it requires $\succ$ to be monotonic on all symbols) and it does not use other DP- resp. DT-based processors.

12 An alternative such example is shown in [8, Ex. 11].

${ }^{13}$ As noted by [22], this can be weakened by replacing $\operatorname{dh}\left(t^{\sharp}, \succ\right)$ with $\operatorname{dh}\left(t^{\sharp}, \succ \cap \stackrel{\mathrm{i}}{\rightarrow}_{\mathcal{D} / \mathcal{R}}\right)$, where $\rightarrow_{\mathcal{D} / \mathcal{R}}=\rightarrow_{\mathcal{R}}^{*} \circ \rightarrow_{\mathcal{D}} \circ \rightarrow_{\mathcal{R}}^{*}$ and $\stackrel{i}{\mathcal{D} / \mathcal{R}}_{\mathcal{R}}$ is the restriction of $\rightarrow_{\mathcal{D} / \mathcal{R}}$ where in each rewrite step with $\rightarrow_{\mathcal{R}}$ or $\rightarrow_{\mathcal{D}}$, the arguments of the redex must be in $(\mathcal{D} \cup \mathcal{R})$-normal form, cf. [3]. Such a weakening is required to use reduction pairs based on path orders where a term $t^{\sharp}$ may start $\succ$-decreasing sequences of arbitrary (finite) length. 
To automate Thm. 23, we need reduction pairs $(\succsim, \succ)$ where an upper bound $c$ for $\iota\left(\operatorname{irc}_{\succ}\right)$ is easy to compute. This holds for reduction pairs based on polynomial interpretations with coefficients from $\mathbb{N}$ (which are well suited for automation). For CoM-monotonicity, we restrict ourselves to complexity polynomial interpretations (CPIs) [.] where $\left[\mathrm{Com}_{n}\right]\left(x_{1}, \ldots, x_{n}\right)=x_{1}+\ldots+x_{n}$ for all $n \in \mathbb{N}$. This is the "smallest" polynomial which is monotonic in $x_{1}, \ldots, x_{n}$. As $\mathrm{CoM}_{n}$ only occurs on right-hand sides of inequalities, $\left[\mathrm{CoM}_{n}\right]$ should be as small as possible.

Moreover, a $C P I$ interprets constructors $f \in \Sigma \backslash \Sigma_{d}$ by polynomials $[f]\left(x_{1}, \ldots\right.$, $\left.x_{n}\right)=a_{1} x_{1}+\ldots+a_{n} x_{n}+b$ where $b \in \mathbb{N}$ and $a_{i} \in\{0,1\}$. This ensures that the mapping from constructor ground terms $t \in \mathcal{T}\left(\Sigma \backslash \Sigma_{d}, \varnothing\right)$ to their interpretations is in $\mathcal{O}(|t|)$, cf. $[7,17]$. Note that the interpretations in Ex. 22 were CPIs.

Thm. 24 shows how such interpretations can be used ${ }^{14}$ for the processor of Thm. 23. Here, as an upper bound $c$ for $\iota\left(\operatorname{irc}_{\succ}\right)$, one can simply take $\mathcal{P o l}_{m}$, where $m$ is the maximal degree of the polynomials in the interpretation.

Theorem 24 (Reduction Pair Processor with Polynomial Interpretations). Let $P=\langle\mathcal{D}, \mathcal{S}, \mathcal{R}\rangle$ be a $D T$ problem and let $\succsim$ and $\succ$ be induced by a $C P I[\cdot]$. Let $m \in \mathbb{N}$ be the maximal degree of all polynomials $\left[f^{\sharp}\right]$, for all $f^{\sharp}$ with $f \in \Sigma_{d}$. Let $\mathcal{D} \subseteq \succsim \cup \succ$ and $\mathcal{R} \subseteq \succsim$. Then the following processor is sound: $\operatorname{Proc}(\langle\mathcal{D}, \mathcal{S}, \mathcal{R}\rangle)=\left(\mathcal{P o l}_{m},\left\langle\mathcal{D}, \mathcal{S} \backslash \mathcal{D}_{\succ}, \mathcal{R}\right\rangle\right)$.

Example 25. This TRS [1] illustrates Thm. 24, where $\mathbf{q}(x, y, y)$ computes $\left\lfloor\frac{x}{y}\right\rfloor$.

$\mathrm{q}(0, \mathrm{~s}(y), \mathrm{s}(z)) \rightarrow 0 \quad \mathrm{q}(\mathrm{s}(x), \mathrm{s}(y), z) \rightarrow \mathrm{q}(x, y, z) \quad \mathrm{q}(x, 0, \mathrm{~s}(z)) \rightarrow \mathrm{s}(\mathrm{q}(x, \mathrm{~s}(z), \mathrm{s}(z)))$

The dependency tuples $\mathcal{D}$ of this TRS are

$$
\begin{aligned}
\mathrm{q}^{\sharp}(0, \mathrm{~s}(y), \mathbf{s}(z)) \rightarrow \text { Com }_{0} \quad(9) \quad \mathrm{q}^{\sharp}(\mathbf{s}(x), \mathbf{s}(y), z) & \rightarrow \operatorname{Com}_{1}\left(\mathbf{q}^{\sharp}(x, y, z)\right) \\
\mathbf{q}^{\sharp}(x, 0, \mathbf{s}(z)) & \rightarrow \operatorname{Com}_{1}\left(\mathbf{q}^{\sharp}(x, \mathbf{s}(z), \mathbf{s}(z))\right)
\end{aligned}
$$

As the usable rules are empty, Thm. 20 transforms the canonical DT problem to $\langle\mathcal{D}, \mathcal{D}, \varnothing\rangle$. Consider the CPI $[0]=0,[\mathrm{~s}](x)=x+1,\left[\mathbf{q}^{\sharp}\right](x, y, z)=x+1,\left[\mathrm{COM}_{0}\right]=$ $0,\left[\mathrm{Com}_{1}\right](x)=x$. With the corresponding reduction pair, the DTs (9) and (10) are strictly decreasing and (11) is weakly decreasing. Moreover, the degree of $\left[q^{\sharp}\right]$ is 1 . Hence, the reduction pair processor returns $\left(\mathcal{P o l}_{1},\langle\mathcal{D},\{(11)\}, \varnothing\rangle\right)$. Unfortunately, no reduction pair based on CPIs orients (11) strictly and both (9) and (10) weakly. So for the moment we cannot simplify this problem further.

\subsection{Dependency Graph Processors}

As in the DP framework for termination, it is useful to have a finite representation of (a superset of) all possible chain trees.

\footnotetext{
${ }^{14}$ Alternatively, our reduction pair processor can also use matrix interpretations $[8,19$, $21,23,27]$, polynomial path orders $\left(\mathrm{POP}^{*}[3]\right)$, etc. For POP*, we would extend $\mathfrak{C}$ by a complexity $\mathcal{P o l}_{*}$ for polytime computability, where $\mathcal{P}_{0} l_{n} \sqsubset \mathcal{P} l_{*} \sqsubset$ ? for all $n \in \mathbb{N}$.
} 
Definition 26 (Dependency Graph). Let $\mathcal{D}$ be a set of DTs and $\mathcal{R}$ a TRS. The $(\mathcal{D}, \mathcal{R})$-dependency graph is the directed graph whose nodes are the DTs in $\mathcal{D}$ and there is an edge from $s \rightarrow t$ to $u \rightarrow v$ in the dependency graph iff there is a chain tree with an edge from a node $\left(s \rightarrow t \mid \sigma_{1}\right)$ to a node $\left(u \rightarrow v \mid \sigma_{2}\right)$.

Every $(\mathcal{D}, \mathcal{R})$-chain corresponds to a path in the $(\mathcal{D}, \mathcal{R})$-dependency graph. While dependency graphs are not computable in general, there are several techniques to compute over-approximations of dependency graphs for termination, cf. e.g. [1]. These techniques can also be applied for $(\mathcal{D}, \mathcal{R})$-dependency graphs.

Example 2\%. For the TRS $\mathcal{R}$ from Ex. 3, we obtain the following $\left(\mathcal{D}, \mathcal{R}_{1}\right)$ dependency graph, where $\mathcal{D}=D T(\mathcal{R})$ and $\mathcal{R}_{1}$ are the gt- and p-rules.

$$
\begin{aligned}
& \text { Q } \\
& \mathrm{gt}^{\sharp}(0, k) \rightarrow \mathrm{CoM}_{0}(6) \longleftarrow \mathrm{gt}^{\sharp}(\mathrm{s}(n), \mathrm{s}(k)) \rightarrow \mathrm{CoM}_{1}\left(\mathrm{gt}^{\sharp}(n, k)\right)(8)
\end{aligned}
$$

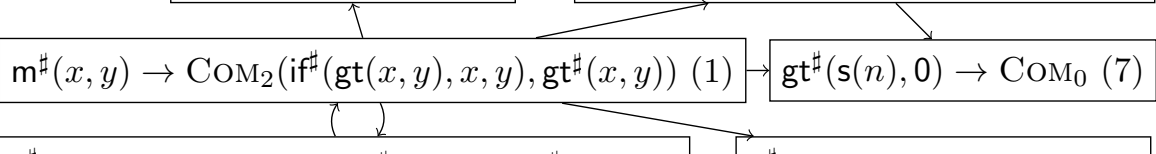

$$
\begin{aligned}
& \text { if } \left.{ }^{\sharp}(\text { true }, x, y) \rightarrow \mathrm{Com}_{2}\left(\mathrm{~m}^{\sharp}(\mathrm{p}(x), y), \mathrm{p}^{\sharp}(x)\right)(2) \quad \text { if }{ }^{\sharp} \text { false, } x, y\right) \rightarrow \mathrm{CoM}_{0}(3) \\
& \mathrm{p}^{\sharp}(0) \rightarrow \mathrm{Com}_{0}(4) \quad \mathrm{p}^{\sharp}(\mathrm{s}(n)) \rightarrow \mathrm{Com}_{0}(5)
\end{aligned}
$$

For termination analysis, one can regard strongly connected components of the graph separately and ignore nodes that are not on cycles. This is not possible for complexity analysis: If one regards the DTs $\mathcal{D}^{\prime}=\{(1),(2)\}$ and $\mathcal{D}^{\prime \prime}=\{(8)\}$ on the two cycles of the graph separately, then both resulting DT problems $\left\langle\mathcal{D}^{\prime}, \mathcal{D}^{\prime}, \mathcal{R}_{1}\right\rangle$ and $\left\langle\mathcal{D}^{\prime \prime}, \mathcal{D}^{\prime \prime}, \mathcal{R}_{1}\right\rangle$ have linear complexity. However, this allows no conclusions on the complexity of $\left\langle\mathcal{D}, \mathcal{D}, \mathcal{R}_{1}\right\rangle$ (which is quadratic). Nevertheless, it is possible to remove DTs $s \rightarrow t$ that are leaves (i.e., $s \rightarrow t$ has no successors in the dependency graph). This yields $\left\langle\mathcal{D}_{1}, \mathcal{D}_{1}, \mathcal{R}_{1}\right\rangle$, where $\mathcal{D}_{1}=\{(1),(2),(8)\}$.

Theorem 28 (Leaf Removal Processor). Let $\langle\mathcal{D}, \mathcal{S}, \mathcal{R}\rangle$ be a DT problem and let $s \rightarrow t \in \mathcal{D}$ be a leaf in the $(\mathcal{D}, \mathcal{R})$-dependency graph. Then the following processor is sound: $\operatorname{Proc}(\langle\mathcal{D}, \mathcal{S}, \mathcal{R}\rangle)=\left(\mathcal{P}_{0} l_{0},\langle\mathcal{D} \backslash\{s \rightarrow t\}, \mathcal{S} \backslash\{s \rightarrow t\}, \mathcal{R}\rangle\right)$.

\subsection{Knowledge Propagation}

In the DP framework for termination, the reduction pair processor removes "strictly decreasing" DPs. While this is unsound for complexity analysis (cf. Ex. 22), we now show that by an appropriate extension of DT problems, one can obtain a similar processor also for complexity analysis.

Lemma 29 shows that we can estimate the complexity of a DT if we know the complexity of all its predecessors in the dependency graph.

Lemma 29 (Complexity Bounded by Predecessors). Let $\langle\mathcal{D}, \mathcal{S}, \mathcal{R}\rangle$ be a $D T$ problem and $s \rightarrow t \in \mathcal{D}$. Let Pre $(s \rightarrow t) \subseteq \mathcal{D}$ be the predecessors of $s \rightarrow t$, i.e., Pre $(s \rightarrow t)$ contains all DTs $u \rightarrow v$ where there is an edge from $u \rightarrow v$ to $s \rightarrow t$ in the $(\mathcal{D}, \mathcal{R})$-dependency graph. Then $\iota_{\langle\mathcal{D},\{s \rightarrow t\}, \mathcal{R}\rangle} \sqsubseteq \iota_{\langle\mathcal{D}, \operatorname{Pre}(s \rightarrow t), \mathcal{R}\rangle}$. 
Example 30. Consider the TRS from Ex. 25. By usable rules and reduction pairs, we obtained $\langle\mathcal{D},\{(11)\}, \varnothing\rangle$ for $\mathcal{D}=$ $\{(9),(10),(11)\}$. The leaf re-

$$
\begin{aligned}
& \Omega \\
& \mathrm{q}^{\sharp}(\mathrm{s}(x), \mathrm{s}(y), z) \rightarrow \operatorname{Com}_{1}\left(\mathrm{q}^{\sharp}(x, y, z)\right)(10) \\
& \mathrm{q}^{\sharp}(x, 0, \mathrm{~s}(z)) \rightarrow \operatorname{Com}_{1}\left(\mathrm{q}^{\sharp}(x, \mathrm{~s}(z), \mathrm{s}(z))\right)(11)
\end{aligned}
$$
moval processor yields $\left\langle\mathcal{D}^{\prime},\{(11)\}, \varnothing\right\rangle$ with $\mathcal{D}^{\prime}=\{(10),(11)\}$. Consider the the $\left(\mathcal{D}^{\prime}, \varnothing\right)$-dependency graph above. We have $\iota_{\left\langle\mathcal{D}^{\prime},\{(11)\}, \varnothing\right\rangle} \sqsubseteq \iota_{\left\langle\mathcal{D}^{\prime},\{(10)\}, \varnothing\right\rangle}$ by Lemma 29, since (10) is the only predecessor of (11). Thus, the complexity of $\left\langle\mathcal{D}^{\prime},\{(11)\}, \varnothing\right\rangle$ does not matter for the overall complexity, if we can guarantee that we have already taken the complexity of $\left\langle\mathcal{D}^{\prime},\{(10)\}, \varnothing\right\rangle$ into account.

Therefore, we now extend the definition of DT problems by a set $\mathcal{K}$ of DTs with "known" complexity, i.e., the complexity of the DTs in $\mathcal{K}$ has already been taken into account. So a processor only needs to estimate the complexity of a set of DTs correctly if their complexity is higher than the complexity of the DTs in $\mathcal{K}$. Otherwise, the processor may return an arbitrary result. To this end, we introduce a "subtraction" operation $\ominus$ on complexities from $\mathfrak{C}$.

Definition 31 (Extended DT Problems, $\Theta$ ). For $c, d, \in \mathfrak{C}$, let $c \ominus d=c$ if $d \sqsubset c$ and $c \ominus d=\mathcal{P}_{0} l_{0}$ if $c \sqsubseteq d$. Let $\mathcal{R}$ be a TRS, $\mathcal{D}$ a set of DTs, and $\mathcal{S}, \mathcal{K} \subseteq \mathcal{D}$. Then $\langle\mathcal{D}, \mathcal{S}, \mathcal{K}, \mathcal{R}\rangle$ is an extended DT problem and $\langle D T(\mathcal{R}), D T(\mathcal{R}), \varnothing, \mathcal{R}\rangle$ is the canonical extended DT problem for $\mathcal{R}$. We define the complexity of an extended DT problem to be $\gamma_{\langle\mathcal{D}, \mathcal{S}, \mathcal{K}, \mathcal{R}\rangle}=\iota_{\langle\mathcal{D}, \mathcal{S}, \mathcal{R}\rangle} \ominus \iota_{\langle\mathcal{D}, \mathcal{K}, \mathcal{R}\rangle}$ and also use $\gamma$ instead of $\iota$ in the soundness condition for processors. So on extended DT problems, a processor with $\operatorname{Proc}(P)=\left(c, P^{\prime}\right)$ is sound if $\gamma_{P} \sqsubseteq c \oplus \gamma_{P^{\prime}}$. An extended DT problem $\langle\mathcal{D}, \mathcal{S}, \mathcal{K}, \mathcal{R}\rangle$ is solved if $\mathcal{S}=\varnothing$.

So for $\mathcal{K}=\varnothing$, the definition of "complexity" for extended DT problems is equivalent to complexity for ordinary DT problems, i.e., $\gamma_{\langle\mathcal{D}, \mathcal{S}, \varnothing, \mathcal{R}\rangle}=\iota_{\langle\mathcal{D}, \mathcal{S}, \mathcal{R}\rangle}$. Cor. 32 shows that our approach is still correct for extended DT problems.

Corollary 32 (Correctness). If $P_{0}$ is the canonical extended DT problem for $a T R S \mathcal{R}$ and $P_{0} \stackrel{c_{1}}{\sim} \ldots \stackrel{c_{k}}{\sim} P_{k}$ is a proof chain, then $\iota_{\mathcal{R}}=\gamma_{P_{0}} \sqsubseteq c_{1} \oplus \ldots \oplus c_{k}$.

Now we introduce a processor which makes use of $\mathcal{K}$. It moves a DT $s \rightarrow t$ from $\mathcal{S}$ to $\mathcal{K}$ whenever the complexity of all predecessors of $s \rightarrow t$ in the dependency graph has already been taken into account. ${ }^{15}$

Theorem 33 (Knowledge Propagation Processor). Let $\langle\mathcal{D}, \mathcal{S}, \mathcal{K}, \mathcal{R}\rangle$ be an extended DT problem, $s \rightarrow t \in \mathcal{S}$, and Pre $(s \rightarrow t) \subseteq \mathcal{K}$. Then the following processor is sound: $\operatorname{PrOC}(\langle\mathcal{D}, \mathcal{S}, \mathcal{K}, \mathcal{R}\rangle)=\left(\mathcal{P} o l_{0},\langle\mathcal{D}, \mathcal{S} \backslash\{s \rightarrow t\}, \mathcal{K} \cup\{s \rightarrow t\}, \mathcal{R}\rangle\right)$.

Before we can illustrate this processor, we need to adapt the previous processors to extended DT problems. The adaption of the usable rules and leaf removal processors is straightforward. But now the reduction pair processor does not only delete DTs from $\mathcal{S}$, but moves them to $\mathcal{K}$. The reason is that the complexity of these DTs is bounded by the complexity value $c \in \mathfrak{C}$ returned by the processor. (Of course, the special case of the reduction pair processor with polynomial

${ }^{15}$ In particular, this means that nodes without predecessors (i.e., "roots" of the dependency graph that are not in any cycle) can always be moved from $\mathcal{S}$ to $\mathcal{K}$. 
interpretations of Thm. 24 can be adapted analogously.)

Theorem 34 (Processors for Extended DT Problems). Let $P=\langle\mathcal{D}, \mathcal{S}$, $\mathcal{K}, \mathcal{R}\rangle$ be an extended DT problem. Then the following processors are sound.

- The usable rules processor: $\operatorname{Proc}(P)=\left(\mathcal{P o l}_{0},\left\langle\mathcal{D}, \mathcal{S}, \mathcal{K}, \mathcal{U}_{\mathcal{R}}(\mathcal{D})\right\rangle\right)$.

- The leaf removal processor $\operatorname{Proc}(P)=\left(\mathcal{P}_{0} l_{0},\langle\mathcal{D} \backslash\{s \rightarrow t\}, \mathcal{S} \backslash\{s \rightarrow t\}\right.$, $\mathcal{K} \backslash\{s \rightarrow t\}, \mathcal{R}\rangle)$, if $s \rightarrow t$ is a leaf in the $(\mathcal{D}, \mathcal{R})$-dependency graph.

- The reduction pair processor: $\operatorname{PROC}(P)=\left(c,\left\langle\mathcal{D}, \mathcal{S} \backslash \mathcal{D}_{\succ}, \mathcal{K} \cup \mathcal{D}_{\succ}, \mathcal{R}\right\rangle\right)$, if $(\succsim, \succ)$ is a Com-monotonic reduction pair, $\mathcal{D} \subseteq \succsim \cup \succ, \mathcal{R} \subseteq \succsim$, and $c \sqsupseteq \iota\left(\operatorname{irc}_{\succ}\right)$ for the function $\operatorname{irc}_{\succ}(n)=\sup \left\{\operatorname{dh}\left(t^{\sharp}, \succ\right)\left|t \in \mathcal{T}_{B},\right| t \mid \leq n\right\}$.

Example 35. Reconsider the TRS $\mathcal{R}$ for division from Ex. 25. Starting with its canonical extended DT problem, we now obtain the following proof chain.

$\begin{array}{lllll}\langle\{(9),(10),(11)\}, & \{(9),(10),(11)\}, \varnothing, & \mathcal{R}\rangle & \\ \stackrel{\mathcal{P}_{\text {ol }} l_{0}}{\sim}\langle\{(10),(11)\}, & \{(10),(11)\}, & \varnothing, & \mathcal{R}\rangle & \text { (leaf removal) } \\ \stackrel{\mathcal{P}_{0} l_{0}}{\sim}\langle\{(10),(11)\}, & \{(10),(11)\}, & \varnothing, & \varnothing\rangle & \text { (usable rules) } \\ \stackrel{\mathcal{P}_{0} l_{1}}{\sim}\langle\{(10),(11)\}, & \{(11)\}, & \{(10)\}, & \varnothing\rangle & \text { (reduction pair) } \\ \stackrel{\mathcal{P}_{0} l_{0}}{\sim}\langle\{(10),(11)\}, & \varnothing, & \{(10),(11)\}, \varnothing\rangle & \text { (knowledge propag.) }\end{array}$

For the last step we use Pre $((11))=\{(10)\}$, cf. Ex. 30. The last DT problem is solved. Thus, $\iota_{\mathcal{R}} \sqsubseteq \mathcal{P} l_{0} \oplus \mathcal{P}_{0} l_{0} \oplus \mathcal{P}_{o} l_{1} \oplus \mathcal{P o l}_{0}=\mathcal{P} l_{1}$, i.e., $\mathcal{R}$ has linear complexity.

\subsection{Transformation Processors}

To increase power, the DP framework for termination analysis has several processors which transform a DP into new ones (by "narrowing", "rewriting", "instantiation", or "forward instantiation") [11]. We now show how to adapt such processors for complexity analysis. For reasons of space, we only present the narrowing processor (the other processors can be adapted in a similar way).

For an extended DT problem $\langle\mathcal{D}, \mathcal{S}, \mathcal{K}, \mathcal{R}\rangle$, let $s \rightarrow t \in \mathcal{D}$ with $t=\operatorname{Com}_{n}\left(t_{1}\right.$, $\left.\ldots, t_{i}, \ldots, t_{n}\right)$. If there exists a (variable-renamed) $u \rightarrow v \in \mathcal{D}$ where $t_{i}$ and $u$ have an mgu $\mu$ and both $s \mu$ and $u \mu$ are in $\mathcal{R}$-normal form, then we call $\mu$ a narrowing substitution of $t_{i}$ and define the corresponding narrowing result to be $t_{i} \mu$.

Moreover, if $s \rightarrow t$ has a successor $u \rightarrow v$ in the $(\mathcal{D}, \mathcal{R})$-dependency graph where $t_{i}$ and $u$ have no such mgu, then we obtain additional narrowing substitutions and narrowing results for $t_{i}$. The reason is that in any possible reduction $t_{i} \sigma \stackrel{i}{\rightarrow}_{\mathcal{R}}^{*} u \tau$ in a chain, the term $t_{i} \sigma$ must be rewritten at least one step before it reaches $u \tau$. The idea of the narrowing processor is to already perform this first reduction step directly on the DT $s \rightarrow t$. Whenever a subterm $\left.t_{i}\right|_{\pi} \notin \mathcal{V}$ of $t_{i}$ unifies with the left-hand side of a (variable-renamed) rule $\ell \rightarrow r \in \mathcal{R}$ using an mgu $\mu$ where $s \mu$ is in $\mathcal{R}$-normal form, then $\mu$ is a narrowing substitution of $t_{i}$ and the corresponding narrowing result is $w=t_{i}[r]_{\pi} \mu$.

If $\mu_{1}, \ldots, \mu_{d}$ are all narrowing substitutions of $t_{i}$ with the corresponding narrowing results $w_{1}, \ldots, w_{d}$, then $s \rightarrow t$ can be replaced by $s \mu_{j} \rightarrow \operatorname{Com}_{n}\left(t_{1} \mu_{j}, \ldots\right.$, $\left.t_{i-1} \mu_{j}, w_{j}, t_{i+1} \mu_{j}, \ldots, t_{n} \mu_{j}\right)$ for all $1 \leq j \leq d$. 
However, there could be a $t_{k}$ (with $k \neq i$ ) which was involved in a chain (i.e., $t_{k} \sigma \stackrel{\mathrm{i}}{\rightarrow}_{\mathcal{R}}^{*} u \tau$ for some $u \rightarrow v \in \mathcal{D}$ and some $\sigma, \tau$ ), but this chain is no longer possible when instantiating $t_{k}$ to $t_{k} \mu_{1}, \ldots, t_{k} \mu_{d}$. We say that $t_{k}$ is captured by $\mu_{1}$, $\ldots, \mu_{d}$ if for each narrowing substitution $\rho$ of $t_{k}$, there is a $\mu_{j}$ that is more general (i.e., $\rho=\mu_{j} \rho^{\prime}$ for some substitution $\rho^{\prime}$ ). The narrowing processor has to add another DT $s \rightarrow \operatorname{CoM}_{m}\left(t_{k_{1}}, \ldots, t_{k_{m}}\right)$ where $t_{k_{1}}, \ldots, t_{k_{m}}$ are all terms from $t_{1}$, $\ldots, t_{n}$ which are not captured by the narrowing substitutions $\mu_{1}, \ldots, \mu_{d}$ of $t_{i}$.

This leads to the following processor. For any sets $\mathcal{D}, \mathcal{M}$ of $\mathrm{DTs}, \mathcal{D}[s \rightarrow t / \mathcal{M}]$ denotes the result of replacing $s \rightarrow t$ by the DTs in $\mathcal{M}$. So if $s \rightarrow t \in \mathcal{D}$, then $\mathcal{D}[s \rightarrow t / \mathcal{M}]=(D T \backslash\{s \rightarrow t\}) \cup \mathcal{M}$ and otherwise, $\mathcal{D}[s \rightarrow t / \mathcal{M}]=\mathcal{D}$.

Theorem 36 (Narrowing Processor). Let $P=\langle\mathcal{D}, \mathcal{S}, \mathcal{K}, \mathcal{R}\rangle$ be an extended $D T$ problem and let $s \rightarrow t \in \mathcal{D}$ with $t=\operatorname{Com}_{n}\left(t_{1}, \ldots, t_{i}, \ldots, t_{n}\right)$. Let $\mu_{1}, \ldots, \mu_{d}$ be the narrowing substitutions of $t_{i}$ with the corresponding narrowing results $w_{1}, \ldots, w_{d}$, where $d \geq 0$. Let $t_{k_{1}}, \ldots, t_{k_{m}}$ be the terms from $t_{1}, \ldots, t_{n}$ that are not captured by $\mu_{1}, \ldots, \mu_{d}$, where $k_{1}, \ldots, k_{m}$ are pairwise different. We define

$$
\begin{aligned}
& \mathcal{M}=\left\{s \mu_{j} \rightarrow \operatorname{CoM}_{n}\left(t_{1} \mu_{j}, \ldots, t_{i-1} \mu_{j}, w_{j}, t_{i+1} \mu_{j}, \ldots, t_{n} \mu_{j}\right) \mid 1 \leq j \leq d\right\} \\
& \cup\left\{s \rightarrow \operatorname{Com}_{m}\left(t_{k_{1}}, \ldots, t_{k_{m}}\right)\right\} .
\end{aligned}
$$

Then the following processor is sound: $\operatorname{PrOC}(P)=\left(\mathcal{P o l}_{0},\left\langle\mathcal{D}^{\prime}, \mathcal{S}^{\prime}, \mathcal{K}^{\prime}, \mathcal{R}\right\rangle\right)$, where $\mathcal{D}^{\prime}=\mathcal{D}[s \rightarrow t / \mathcal{M}]$ and $\mathcal{S}^{\prime}=\mathcal{S}[s \rightarrow t / \mathcal{M}] . \mathcal{K}^{\prime}$ results from $\mathcal{K}$ by removing $s \rightarrow t$ and all DTs that are reachable from $s \rightarrow t$ in the $(\mathcal{D}, \mathcal{R})$-dependency graph. ${ }^{16}$

Example 3\%. To illustrate the narrowing processor, consider the following TRS.

$\mathrm{f}(\mathrm{c}(n, x)) \rightarrow \mathrm{c}(\mathrm{f}(\mathrm{g}(\mathrm{c}(n, x))), \mathrm{f}(\mathrm{h}(\mathrm{c}(n, x)))) \quad \mathrm{g}(\mathrm{c}(0, x)) \rightarrow x \quad \mathrm{~h}(\mathrm{c}(1, x)) \rightarrow x$

So f operates on "lists" of $0 \mathrm{~s}$ and $1 \mathrm{~s}$, where g removes a leading 0 and $\mathrm{h}$ removes a leading 1. Since g's and h's applicability "exclude" each other, the TRS has linear (and not exponential) complexity. The leaf removal and usable rules processors yield the problem $\langle\{(12)\},\{(12)\}, \varnothing,\{\mathrm{g}(\mathrm{c}(0, x)) \rightarrow x, \mathrm{~h}(\mathrm{c}(1, x)) \rightarrow x\}\rangle$ with

$$
\mathrm{f}^{\sharp}(\mathrm{c}(n, x)) \rightarrow \mathrm{COM}_{4}\left(\mathrm{f}^{\sharp}(\mathrm{g}(\mathrm{c}(n, x))), \mathrm{g}^{\sharp}(\mathrm{c}(n, x)), \mathrm{f}^{\sharp}(\mathrm{h}(\mathrm{c}(n, x))), \mathrm{h}^{\sharp}(\mathrm{c}(n, x))\right) \text {. }
$$

The only narrowing substitution of $t_{1}=\mathrm{f}^{\sharp}(\mathrm{g}(\mathrm{c}(n, x)))$ is $[n / 0]$ and the corresponding narrowing result is $\mathrm{f}^{\sharp}(x)$. However, $t_{3}=\mathrm{f}^{\sharp}(\mathrm{h}(\mathrm{c}(n, x)))$ is not captured by the substitution $[n / 0]$, since $[n / 0]$ is not more general than $t_{3}$ 's narrowing substitution $[n / 1]$. Hence, the DT (12) is replaced by the following two new DTs:

$$
\begin{aligned}
& \mathrm{f}^{\sharp}(\mathrm{c}(0, x)) \rightarrow \mathrm{COM}_{4}\left(\mathrm{f}^{\sharp}(x), \mathrm{g}^{\sharp}(\mathrm{c}(0, x)), \mathrm{f}^{\sharp}(\mathrm{h}(\mathrm{c}(0, x))), \mathrm{h}^{\sharp}(\mathrm{c}(0, x))\right) \\
& \mathrm{f}^{\sharp}(\mathrm{c}(n, x)) \rightarrow \mathrm{COM}_{1}\left(\mathrm{f}^{\sharp}(\mathrm{h}(\mathrm{c}(n, x)))\right)
\end{aligned}
$$

Another application of the narrowing processor replaces (14) by $\mathrm{f}^{\sharp}(\mathrm{c}(1, x)) \rightarrow$

\footnotetext{
${ }^{16}$ We cannot define $\mathcal{K}^{\prime}=\mathcal{K}[s \rightarrow t / \mathcal{M}]$, because the narrowing step performed on $s \rightarrow t$ does not necessarily correspond to an innermost reduction. Hence, there can be $\left(\mathcal{D}^{\prime}, \mathcal{R}\right)$-chains that correspond to non-innermost reductions with $\mathcal{D} \cup \mathcal{R}$. So there may exist terms whose maximal $\left(\mathcal{D}^{\prime}, \mathcal{R}\right)$-chain tree is larger than their maximal $(\mathcal{D}, \mathcal{R})$-chain tree and thus, $\iota_{\left\langle\mathcal{D}^{\prime}, \mathcal{K}[s \rightarrow t / \mathcal{M}], \mathcal{R}\right\rangle} \sqsupseteq \iota_{\langle\mathcal{D}, \mathcal{K}, \mathcal{R}\rangle}$. But we need $\iota_{\left\langle\mathcal{D}^{\prime}, \mathcal{K}^{\prime}, \mathcal{R}\right\rangle} \sqsubseteq$ $\iota_{\langle\mathcal{D}, \mathcal{K}, \mathcal{R}\rangle}$ in order to guarantee the soundness of the processor, i.e., to ensure that $\gamma_{\langle\mathcal{D}, \mathcal{S}, \mathcal{K}, \mathcal{R}\rangle}=\iota_{\langle\mathcal{D}, \mathcal{S}, \mathcal{R}\rangle} \ominus \iota_{\langle\mathcal{D}, \mathcal{K}, \mathcal{R}\rangle} \sqsubseteq \iota_{\left\langle\mathcal{D}^{\prime}, \mathcal{S}^{\prime}, \mathcal{R}\right\rangle} \ominus \iota_{\left\langle\mathcal{D}^{\prime}, \mathcal{K}^{\prime}, \mathcal{R}\right\rangle}=\gamma_{\left\langle\mathcal{D}^{\prime}, \mathcal{S}^{\prime}, \mathcal{K}^{\prime}, \mathcal{R}\right\rangle} \cdot$
} 
$\operatorname{Com}_{1}\left(f^{\sharp}(x)\right) \cdot{ }^{17}$ Now $\iota_{\mathcal{R}} \sqsubseteq \mathcal{P o l}_{1}$ is easy to show by the reduction pair processor.

Example 38. Reconsider the TRS of Ex. 3. The canonical extended DT problem is transformed to $\left\langle\mathcal{D}_{1}, \mathcal{D}_{1}, \varnothing, \mathcal{R}_{1}\right\rangle$, where $\mathcal{D}_{1}=\{(1),(2),(8)\}$ and $\mathcal{R}_{1}$ are the gt- and p-rules, cf. Ex. 27. In $\mathrm{m}^{\sharp}(x, y) \rightarrow \mathrm{CoM}_{2}\left(\mathrm{iff}^{\sharp}(\mathrm{gt}(x, y), x, y)\right.$, gt $\left.{ }^{\sharp}(x, y)\right)(1)$, one can narrow $t_{1}=$ if $^{\sharp}(\operatorname{gt}(x, y), x, y)$. Its narrowing substitutions are $[x / 0, y / k]$, $[x / \mathrm{s}(n), y / 0],[x / \mathrm{s}(n), y / \mathrm{s}(k)]$. Note that $t_{2}=\mathrm{gt}^{\sharp}(x, y)$ is captured, as its only narrowing substitution is $[x / \mathrm{s}(n), y / \mathrm{s}(k)]$. So (1) can be replaced by

$$
\begin{aligned}
\mathrm{m}^{\sharp}(0, k) & \rightarrow \mathrm{COM}_{2}\left(\text { if }^{\sharp}(\text { false }, 0, k), \mathrm{gt}^{\sharp}(0, k)\right) \\
\mathrm{m}^{\sharp}(\mathrm{s}(n), 0) & \left.\rightarrow \mathrm{COM}_{2}\left(\text { if }^{\sharp} \text { (true, } \mathrm{s}(n), 0\right), \mathrm{gt}^{\sharp}(\mathrm{s}(n), 0)\right) \\
\mathrm{m}^{\sharp}(\mathrm{s}(n), \mathrm{s}(k)) & \rightarrow \mathrm{COM}_{2}\left(\text { if }^{\sharp}(\mathrm{gt}(n, k), \mathrm{s}(n), \mathrm{s}(k)), \mathrm{gt}^{\sharp}(\mathrm{s}(n), \mathrm{s}(k))\right) \\
\mathrm{m}^{\sharp}(x, y) & \rightarrow \mathrm{COM}_{0}
\end{aligned}
$$

The leaf removal processor deletes (15), (18) and yields $\left\langle\mathcal{D}_{2}, \mathcal{D}_{2}, \varnothing, \mathcal{R}_{1}\right\rangle$ with $\mathcal{D}_{2}=$ $\{(16),(17),(2),(8)\}$. We replace if $f^{\sharp}($ true $, x, y) \rightarrow \mathrm{Com}_{2}\left(\mathrm{~m}^{\sharp}(\mathrm{p}(x), y), \mathrm{p}^{\sharp}(x)\right)(2)$ by

$$
\begin{aligned}
& \text { if } \left.{ }^{\sharp} \text { (true, } 0, y\right) \rightarrow \operatorname{Com}_{2}\left(\mathrm{~m}^{\sharp}(0, y), \mathrm{p}^{\sharp}(0)\right) \\
& \text { if }^{\sharp}(\text { true, } \mathrm{s}(n), y) \rightarrow \mathrm{CoM}_{2}\left(\mathrm{~m}^{\sharp}(n, y), \mathrm{p}^{\sharp}(\mathrm{s}(n))\right)
\end{aligned}
$$

by the narrowing processor. The leaf removal processor deletes (19) and the usable rules processor removes the p-rules from $\mathcal{R}_{1}$. This yields $\left\langle\mathcal{D}_{3}, \mathcal{D}_{3}, \varnothing, \mathcal{R}_{2}\right\rangle$, where $\mathcal{D}_{3}=\{(16),(17),(20),(8)\}$ and $\mathcal{R}_{2}$ are the gt-rules. By the polynomial interpretation $[0]=[$ true $]=[$ false $]=\left[\mathrm{p}^{\sharp}\right](x)=0,[\mathrm{~s}](x)=x+2$, $[\mathrm{gt}](x, y)=\left[\mathrm{gt}^{\sharp}\right](x$, $y)=x,\left[\mathrm{~m}^{\sharp}\right](x, y)=(x+1)^{2}$, [if $\left.\sharp^{\sharp}\right](x, y, z)=y^{2}$, all DTs in $\mathcal{D}_{3}$ are strictly decreasing and all rules in $\mathcal{R}_{2}$ are weakly decreasing. So the reduction pair processor yields $\left\langle\mathcal{D}_{3}, \mathcal{D}_{3}, \varnothing, \mathcal{R}_{2}\right\rangle \stackrel{\mathcal{P o l}_{2}}{\sim}\left\langle\mathcal{D}_{3}, \varnothing, \mathcal{D}_{3}, \mathcal{R}_{2}\right\rangle$. As this DT problem is solved, we obtain $\iota_{\mathcal{R}} \sqsubseteq \mathcal{P o l}_{0} \oplus \ldots \oplus \mathcal{P o l}_{0} \oplus \mathcal{P} l_{2}=\mathcal{P}_{2}$, i.e., $\mathcal{R}$ has quadratic complexity.

\section{Evaluation and Conclusion}

We presented a new technique for innermost runtime complexity analysis by adapting the termination techniques of the DP framework. To this end, we introduced several processors to simplify "DT problems", which gives rise to a flexible and modular framework for automated complexity proofs. Thus, recent advances in termination analysis can now also be used for complexity analysis.

To evaluate our contributions, we implemented them in the termination prover AProVE and compared it with the complexity tools CaT 1.5 [28] and TCT 1.6 [2]. We ran the tools on 1323 TRSs from the Termination Problem Data Base used in the International Termination Competition 2010. ${ }^{18}$ As in the competition, each tool had a timeout of 60 seconds for each example. The left half of the

$\overline{17}$ One can also simplify (13) further by narrowing. Its subterm $\mathrm{g}^{\sharp}(\mathrm{c}(0, x))$ has no narrowing substitutions. This (empty) set of narrowing substitutions captures $\mathrm{f}^{\sharp}(\mathrm{h}(\mathrm{c}(0, x)))$ and $\mathrm{h}^{\sharp}(\mathrm{c}(0, x))$ which have no narrowing substitutions either. Since $\mathrm{f}^{\sharp}(x)$ is not captured, (13) can be transformed into $\mathrm{f}^{\sharp}(\mathrm{c}(0, x)) \rightarrow \mathrm{CoM}_{1}\left(\mathrm{f}^{\sharp}(x)\right)$.

18 See http://www.termination-portal .org/wiki/Termination_Competition. 
table compares CaT and AProVE. For instance, the first row means that AProVE showed constant complexity for 209 examples. On those examples, CaT proved linear complexity in 182 cases and failed in 27 cases. So in the light gray part of the table, AProVE gave more precise results than CaT. In the medium gray part, both tools obtained equal results. In the dark gray part, CaT was more precise than AProVE. Similarly, the right half of the table compares TCT and AProVE.

\begin{tabular}{|c|c|c|c|c|c|c|c|c|c|c|c|c|c|}
\hline & \multicolumn{6}{|c|}{$\mathrm{CaT}$} & \multicolumn{6}{|c|}{ TCT } \\
\hline & & $\mathcal{P}_{\text {ol }}$ & $\mathcal{P o l}_{1}$ & $\mathrm{Pol}_{2}$ & $\mathcal{P o l}_{3}$ & no result & $\sum$ & $\mathcal{P}_{\text {ol }}$ & $\mathcal{P O l}_{1}$ & $\mathcal{P o l}_{2}$ & $\mathcal{P o l}_{3}$ & no result & $\sum$ \\
\hline \multirow{6}{*}{ 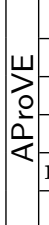 } & $\mathcal{P o l}_{0}$ & - & 182 & - & - & 27 & 209 & 10 & 157 & - & - & 42 & 209 \\
\hline & $\mathcal{P o l}_{1}$ & - & 187 & 7 & - & 76 & 270 & - & 152 & 1 & - & 117 & 270 \\
\hline & $\mathrm{Pol}_{2}$ & - & 32 & 2 & - & 83 & 117 & - & 35 & - & - & 82 & 117 \\
\hline & $\mathcal{P o l}_{3}$ & - & 6 & - & - & 16 & 22 & - & 5 & - & - & 17 & 22 \\
\hline & no result & - & 27 & 3 & 1 & 674 & 705 & - & 22 & 3 & - & 680 & 705 \\
\hline & $\sum$ & 0 & 434 & 12 & 1 & 876 & 1323 & 10 & 371 & 4 & 0 & 938 & 1323 \\
\hline
\end{tabular}

So AProVE showed polynomial innermost runtime for 618 of the 1323 examples $(47 \%)$. (Note that the collection also contains many examples whose complexity is not polynomial.) In contrast, CaT resp. TCT proved polynomial innermost runtime for $447(33 \%)$ resp. $385(29 \%)$ examples. Even a "combined tool" of CaT and TCT (which always returns the better result of these two tools) would only show polynomial runtime for 464 examples (35\%). Hence, our contributions represent a significant advance. This also confirms the results of the Termination Competition 2010, where AProVE won the category of innermost runtime complexity analysis. ${ }^{19}$ AProVE also succeeds on Ex. 3, 25, and 37, whereas CaT and TCT fail. (Ex. 22 can be analyzed by all three tools.) For details on our experiments (including information on the exact DT processors used in each example) and to run our implementation in AProVE via a web interface, we refer to http://aprove.informatik.rwth-aachen.de/eval/RuntimeComplexity/.

Acknowledgments. We are grateful to the CaT and the TCT team for their support with the experiments and to G. Moser and H. Zankl for many helpful comments.

\section{References}

1. T. Arts and J. Giesl. Termination of term rewriting using dependency pairs. Theoretical Computer Science, 236:133-178, 2000.

2. M. Avanzini, G. Moser, and A. Schnabl. Automated implicit computational complexity analysis. In Proc. IJCAR '08, LNAI 5195, pages 132-138, 2008.

3. M. Avanzini and G. Moser. Dependency pairs and polynomial path orders. In Proc. RTA '09, LNCS 5595, pages 48-62, 2009.

4. M. Avanzini and G. Moser. Closing the gap between runtime complexity and polytime computability. In Proc. RTA '10, LIPIcs 6, pages 33-48, 2010.

5. M. Avanzini and G. Moser. Complexity analysis by graph rewriting. In Proc. FLOPS '10, LNCS 6009, pages 257-271, 2010.

6. F. Baader and T. Nipkow. Term Rewriting and All That. Cambridge U. Pr., 1998.

7. G. Bonfante, A. Cichon, J.-Y. Marion, and H. Touzet. Algorithms with polynomial interpretation termination proof. J. Functional Programming, 11(1):33-53, 2001.

${ }^{19}$ In contrast to CaT and TCT, AProVE did not participate in any other complexity categories as it cannot analyze derivational or non-innermost runtime complexity. 
8. J. Endrullis, J. Waldmann, and H. Zantema. Matrix interpretations for proving termination of term rewriting. J. Automated Reasoning, 40(2-3):195-220, 2008.

9. A. Geser, D. Hofbauer, J. Waldmann, and H. Zantema. On tree automata that certify termination of left-linear term rewriting systems. Information and Computation, 205(4):512-534, 2007.

10. J. Giesl, R. Thiemann, P. Schneider-Kamp. The DP framework: Combining techniques for automated termination proofs. LPAR '04, LNAI 3452, p. 301-331, 2005.

11. J. Giesl, R. Thiemann, P. Schneider-Kamp, and S. Falke. Mechanizing and improving dependency pairs. Journal of Automated Reasoning, 37(3):155-203, 2006.

12. J. Giesl, M. Raffelsieper, P. Schneider-Kamp, S. Swiderski, and R. Thiemann. Automated termination proofs for Haskell by term rewriting. ACM Transactions on Programming Languages and Systems, 33(2), 2011.

13. R. Givan and D. A. McAllester. Polynomial-time computation via local inference relations. ACM Transactions on Computational Logic, 3(4):521-541, 2002.

14. N. Hirokawa and A. Middeldorp. Automating the dependency pair method. Information and Computation, 199(1,2):172-199, 2005.

15. N. Hirokawa and G. Moser. Automated complexity analysis based on the dependency pair method. In Proc. IJCAR '08, LNAI 5195, pages 364-379, 2008.

16. N. Hirokawa and G. Moser. Complexity, graphs, and the dependency pair method. In Proc. LPAR '08, LNAI 5330, pages 652-666, 2008.

17. D. Hofbauer and C. Lautemann. Termination proofs and the length of derivations. In Proc. RTA '89, LNCS 355, pages 167-177, 1989.

18. M. Hofmann. Linear types and non-size-increasing polynomial time computation. In Proc. LICS '99, pages 464-473. IEEE Press, 1999.

19. A. Koprowski and J. Waldmann. Max/plus tree automata for termination of term rewriting. Acta Cybernetica, 19(2):357-392, 2009.

20. J.-Y. Marion and R. Péchoux. Characterizations of polynomial complexity classes with a better intensionality. In Proc. PPDP '08, pages 79-88. ACM Press, 2008.

21. G. Moser, A. Schnabl, and J. Waldmann. Complexity analysis of term rewriting based on matrix and context dependent interpretations. In Proc. FSTTCS '08, LIPIcs 2, pages 304-315, 2008.

22. G. Moser. Personal communication, 2010.

23. F. Neurauter, H. Zankl, and A. Middeldorp. Revisiting matrix interpretations for polynomial derivational complexity of term rewriting. In Proc. LPAR '10, LNCS 6397, pages 550-564, 2010.

24. L. Noschinski, F. Emmes, and J. Giesl. A dependency pair framework for innermost complexity analysis of term rewrite systems. Technical Report AIB-2011-03, RWTH Aachen, 2011. Available from http://aib.informatik.rwth-aachen.de.

25. C. Otto, M. Brockschmidt, C. von Essen, J. Giesl. Automated termination analysis of Java Bytecode by term rewriting. In Proc. RTA '10, LIPIcs 6, pp. 259-276, 2010.

26. P. Schneider-Kamp, J. Giesl, T. Ströder, A. Serebrenik, and R. Thiemann. Automated termination analysis for logic programs with cut. Proc. ICLP '10, Theory and Practice of Logic Programming, 10(4-6):365-381, 2010.

27. J. Waldmann. Polynomially bounded matrix interpretations. In Proc. RTA '10, LIPIcs 6, pages 357-372, 2010.

28. H. Zankl and M. Korp. Modular complexity analysis via relative complexity. In Proc. RTA '10, LIPIcs 6, pages 385-400, 2010. 